\title{
Portal vein thrombosis as a rare cause of abdominal pain: When to consider?
}

\author{
Cengiz Tavusbay, Erdinç Kamer, Turan Acar, İbrahim Kokulu, Haldun Kar, Özlem Gür
}

\begin{abstract}
Extrahepatic portal vein thrombosis (PVT) is a rare condition that is characterized by the presence of thrombus within any segment of the portal vein, including the right and left intrahepatic branches. It may also extend to the splenic or superior mesenteric veins. Portal vein thrombosis may be related to cirrhosis or liver malignancy as well as to local inflammatory conditions in the abdomen and genetic or acquired thrombophilic diseases. Currently, PVT is being increasingly diagnosed due to advances in modern imaging techniques. The clinical presentation has a wide range, from an asymptomatic lesion to a potentially life-threatening situation. In this study, we present three patients with PVT. The diagnosis was made by radiologic and clinical findings. In the first patient, genetic testing revealed factor V Leiden mutation as the cause of PVT. The second patient was diagnosed with lupus anticoagulant syndrome as the cause of PVT. Portal vein thrombosis was associated with intra abdominal infection due to anastomotic leakage in the third patient. Two patients were successfully treated with anticoagulant therapy. This report emphasizes that even though PVT is a rare cause of abdominal pain, timely diagnosis and appropriate management is vital due to its lethal complications such as mesenteric ischemia and mesenteric infarct.

Keywords: Anti coagulation treatment, factor $\mathrm{V}$ Leiden mutation, portal vein thrombosis
\end{abstract}

Cite this paper as: Tavusbay C, Kamer E, Acar T, Kokulu I, Kar H, Gür Ö. Portal vein thrombosis as a rare cause of abdominal pain: When to consider? Turk J Surg 2017; 33(2): 126-129

Clinic of General Surgery, Izmir Katip Çelebi University Atatürk Training and Research Hospital, İzmir Turkey

Address for Correspondence
Turan Acar
e-mail: drturanacar1982@
gmail.com
Received: 30.09 .2014
Accepted: 15.12 .2014
Available Online Date: 02.07 .2015
OCopyright 2017
by Turkish Surgical Association
Available online at
www.turkjsurg.com

\section{INTRODUCTION}

Portal vein thrombosis (PVT) was first reported in a patient with liver cirrhosis, with detection of phlebothrombosis in the portal system, in 1869 (1). Portal vein thrombosis is a rare condition characterized by presence of luminal partial or complete thrombosis in any segment of the portal vein including the left intrahepatic branches. It may even spread to the splenic or superior mesenteric veins. Currently, portal vein thrombosis is detected more often owing to the improvements in modern imaging techniques. Its clinical spectrum varies from asymptomatic presentation to life-threatening conditions. Patients may present with various clinical manifestations ranging from vague abdominal pain to sepsis resulting from ischemic necrosis-associated perforation. It may be associated with cirrhosis or liver malignancy, as well as with local inflammatory conditions of the abdomen, and congenital or acquired prothrombotic diseases. Timely and proper interventions reduce mortality and morbidity rates associated with PVT $(1,2)$.

This study aimed to review the clinical characteristics and treatment of PVT based on three patients, each with different clinical presentations, along with a literature review.

\section{CASE PRESENTATIONS}

Case 1. A 53-year-old female patient. She complained of mild abdominal pain for the last three days. Her family history was insignificant. She has been diagnosed with Diabetes Mellitus about 1 month ago. There was no pathological finding on her physical examination except minimal sensitivity on the right upper quadrant of the abdomen. The computed tomography (CT) and abdominal color doppler ultrasonography revealed thrombus in the main portal vein and its intrahepatic branches. Further examination of the patient showed Factor 5 Leiden mutation (FVL). The patient was started on subcutaneous bid $0.6 \mathrm{~mL}$ of low molecular weight heparin (LMWH) with a diagnosis of PVT, which was shifted to oral anticoagulation. The general condition of the patient is good and she is currently being followed-up in the outpatient clinics.

Case 2. A 16-year-old male patient. An incidental appendectomy operation has been performed 10 days prior to admission in our hospital. The operation has been performed in another hospital where he presented with complaints of abdominal pain, nausea and vomiting, with a preliminary diagnosis of acute abdomen, and he underwent appendectomy since there was no pathologic finding except intraabdominal free fluid. The patient was referred to our clinic for further evaluation since his complaints continued. On physical examination, there was non-specific sensitivity in the abdomen and no sign of acute abdomen. 
The pathologic laboratory values included a white blood cell count of 13.100/ul (3.5-10), hematocrit level of 33\%, and creatinine kinase of $1706 \mathrm{U} / \mathrm{L}$ (30-200). Computed tomography and doppler ultrasonography revealed PVT, large collateral veins at the level of the porta hepatis and diffuse peritoneal fluid in the abdominal cavity (Figure 1, 2). Lupus anticoagulant antibodies were found to be elevated in further evaluation. The patient was started on LMWH. He is currently on oral anticoagulation without any problems.

Case 3. A 64-year-old male patient. The patient has undergone an emergency operation 20 days ago in another clinic due to acute abdomen and received partial small bowel resection and end-to-end anastomosis due to small bowel necrosis associated with mesenteric ischemia. In the postoperative period, the patient was admitted to our emergency clinic due to biliary drainage from the abdominal drain. On physical examination, he had extensive brown rashes throughout his body as well as numerous fibromas. The computed tomography showed bilateral pleural effusion, an appearance compatible with thrombosis in the portal vein, diffuse peritoneal free fluid, and splenic infarcts. Anti-thrombolytic therapy was initiated. A laparotomy was performed after the patient's evaluations and resuscitation were completed. On laparotomy, intense adhesions related to the previous operation was detected within the abdomen along with severe edema on the intestinal walls and the peritoneum. Any site of leakage was not observed.

The general condition of the patient improved in the early postoperative period. He tolerated oral diet. On the $6^{\text {th }}$ postoperative day, however, he developed convulsions and sud-

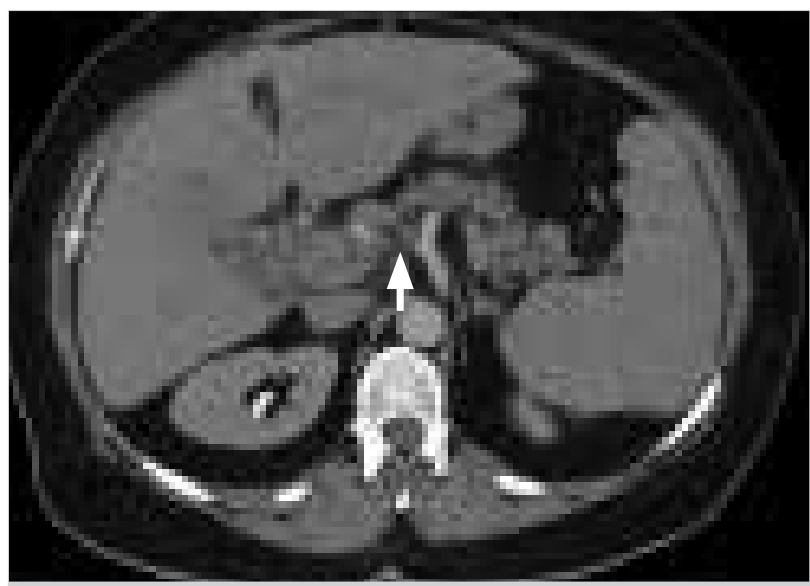

Figure 1. Portal vein thrombosis den respiratory arrest. The patient was immediately intubated and transferred to the intensive care unit. Cranial magnetic resonance imaging (MRI) and $\mathrm{CT}$ revealed an appearance consistent with intracranial plexiform neurofibromas (Neurofibromatosis type 1). As his medical treatment continued, the general condition of the patient deteriorated mainly due to his neurological status and he died at the $46^{\text {th }}$ day of follow-up.

All patients were informed in detail about the disease and the procedures to be performed, and a patient informed consent form was obtained from all. The required approvals were obtained from patients in order to carry out scientific studies.

\section{DISCUSSION}

The likelihood of developing PVT throughout life is around 1\% in the general population (1). In patients with cirrhosis, this incidence is reported to be between $0.6-16 \%$ according to the severity of cirrhosis and the incidence is even higher in patients requiring liver transplantation. The incidence is $10-40 \%$ in patients with liver cancer (1). As seen in Table 1 various factors can cause PVT (1-3). The rate of patients in whom no etiologic factor can be detected, who are accepted as idiopathic, is stated as $8-15 \%$ (1).

Clinical findings vary from patient to patient and are usually non-specific. Although there is a tradition to classify portal vein thrombosis as acute and chronic, it is not always clinically possible to differentiate between the two. Some authors define PVT as acute if the initial clinical manifestations had begun 60 days prior to diagnosis, but this opinion is not universally accepted (1). Acute and chronic PVT are defined as sequential stages of the same disease that occur for similar reasons, but their clinical treatments differ. Acute septic PVT, also called pylephlebitis, is characterized by the presence of infected thrombosis in the portal vein. Usually there is a septic focus in the abdomen. Acute PVT is often asymptomatic or manifests with mild pain, and is generally a coincidental finding on abdominal imaging performed for other causes. However, SMV and mesenteric arc involvement can present with hematochezia due to congestion and ischemia in the intestine depending on the severity of involvement, or can manifest as multiple organ failure ranging from shock, sepsis and even death. Chronic PVT can be asymptomatic and can be detected incidentally on imaging methods. Collateral development (portal cavernous) occurs around the thrombotic portal vein. In these patients, signs of portal hypertension such as splenomegaly, esophageal varices, anemia, and thrombocytopenia can be detected. Upper Gl bleeding can be the first symptom in

Table 1. Etiology of portal vein thrombosis (PVT)

\section{Local factors}

\section{Cirrhosis}

2. Abdominal organ malignant diseases; liver and pancreas primarily

3. Infection and inflammation; pancreatitis, diverticulitis, cholecystitis, appendicitis, neonatal omphalitis etc.

4. Portal vein injury; abdominal surgery, blunt trauma, surgical shunts and transjugular intrahepatic porto-systemic shunt, splenectomy and liver transplantation, etc

\section{Pro-thrombotic factors}

\section{Genetic factors}

a) Mutation; Factor V Leiden, Factor II

b) Deficiencies: Protein C, Protein S deficiency, Antithrombin deficiency

\section{Acquired factors:}

Myeloproliferative diseases, antiphospolipid syndrome, paroxysmal nocturnal hemoglobinuria, pregnancy, oral contraceptive pills, hyperhomocysteinemia, malignancy

3. Idiopathic $10-30 \%$ 
$20-40 \%$ of cases. The prognosis in chronic cases is largely dependent on the presence of underlying cirrhosis or malignancy. The prognosis of portal vein thrombosis in patients without cirrhosis or malignant disease is better than the others. The clinical significance of incidentally detected PVT is unclear.

Imaging methods used for the diagnosis of portal vein thrombosis include Doppler US, abdominal CT and MR. The specificity and sensitivity of Doppler US is reported to be $80-100 \%$ $(3,4)$. Endoscopic ultrasonography was reported to be $81 \%$ sensitive and $93 \%$ specific for the diagnosis of portal vein thrombosis, and may aid in identifying small thrombi which do not produce complete thrombosis (4). Patients with portal vein thrombosis may have normal liver function tests as well as other laboratory tests, except cirrhotic patients. A slight decrease in prothrombin and other coagulation factors, and an increase in D-dimer levels may be detected. Laboratory findings are mostly nonspecific as clinical findings, nevertheless, laboratory findings of any disease and/or genetic disorder associated with PVT can be determined.

In our first case, a factor $\mathrm{V}$ Leiden (FVL) heterozygote mutation was detected. FVL mutation, one of the rare causes of portal venous thrombosis, is a hereditary clotting disorder resulting from the substitution of arginine with glutamine at position 506 in the Factor V gene. It is detected in $3-12 \%$ of healthy individuals worldwide, and in $9 \%$ of healthy individuals in our country. Portal vein thrombosis is common among these patients (5). Some authors suggest systemic screening for the presence of thrombotic factors in patients with a history of portal venous thrombosis, even if local causes are present $(3,6)$.

In our second patient, antiphospholipid syndrome was detected. Antiphospholipid syndrome (antiphospholipid antibody syndrome, APS) or Hughes syndrome is an autoimmune disorder characterized by coagulation disorders caused by antibodies to plasma proteins bound to phospholipids or anionic phospholipids. Antiphospholipid antibodies are a heterogeneous group of antibodies against negatively charged phospholipids, mainly anticardiolipin antibody and lupus anticoagulant antibody as well as phosphatidyl serine, phosphatidyl inositol and phosphatidic acid, and against neutral phosphatidyl etonalamine. Although hypercoagulability resulting from the presence of antiphospholipid antibodies could manifest only as a disorder in coagulation tests, it may also present with cerebrovascular events, venous thrombosis, arterial thrombo- sis, or obstetric complications. APS is a risk factor for portal venous thrombosis in non-cirrhotic patients (7).

Type 1 neurofibromatosis (NF-Type 1, Von Recklinghausen's disease) was detected in the third patient. The disease is autosomal dominant. Involvement of the skin, bone, soft tissue, arterial system and nervous system is accompanied by multiple sclerosis and malignancy development in various organs and tissues of the body. Association with APS that causes thrombotic disorders has also been reported (8).

In this study, different preliminary diagnoses were made clinically in 3 patients, and the diagnosis of PVT could be made by imaging methods. This indicates that the diagnosis can be easily missed in daily clinical practice. For this reason, PVT should be kept in mind especially in patients with vague abdominal pain. It has been reported that in cases with portal vein thrombosis, the primary etiology can be identified in a significant portion of the patients (70\%) by advanced etiopathogenetic tests (7). The aim of treatment in acute PVT is to prevent further progression of thrombosis in the portal venous system and to treat complications associated with PVT (Table 2). There are few randomized studies on this issue. For this reason, the treatment should be individualized. Portal vein thrombosis may spontaneously resolve. However, it is crucial to implement treatment methods for portal vein thrombosis before serious complications arise. Treatment options in these patients include anticoagulant therapy, surgical thrombectomy, endovascular thrombectomy, and thrombolytic therapy. It has been reported that portal vein thrombus was recanalized with anticoagulant therapy in more than $80 \%$ of patients (3). Thrombolytic therapy can also be used, but there are reservations about the use of systemic thrombolytics.

\section{CONCLUSION}

In conclusion, PVT should be considered as part of differential diagnosis in non-specific abdominal pain. In particular, it should be considered in the differential diagnosis of unexplained abdominal pain in patients with intra-abdominal infection and those with previous abdominal surgery including laparoscopic procedures, and both radiologic and laboratory methods should be used for diagnosis. We emphasize the need for timely diagnosis and appropriate treatment of PVT, since although it is a rare cause of abdominal pain it may present with lethal complications such as mesenteric ischemia and infarction.

Table 2. PVT treatment (Based on AASLD)

\section{Acute PVT}

All patients should receive anticoagulation therapy for at least 3 months.

Treatment is started with low molecular weight heparin (LMWH).

Treatment is shifted to oral anticoagulation once the patient has stabilized and if no invasive procedure is planned.

Long term anticoagulation is considered

- in patients with permanent thrombotic risk factors that are not otherwise correctable,

- in patients without a contraindication and thrombus extension into mesenteric veins.

Antibiotics are initiated if there is any evidence of infection.

\section{Chronic PVT}

All patients should be screened for esophageal varices.

Treatment should be applied for active variceal hemorrhage and for primary and secondary prophylaxis according to guidelines for cirrhotic patients.

Long term anticoagulation is considered

in patients without cirrhosis and with permanent thrombotic risk factors that are not otherwise correctable if there is no contraindication.

In patients with gastroesophageal varices, adequate prophylaxis for variceal bleeding should be started before anticoagulation treatment. 
Informed Consent: Written informed consent was obtained from patients who participated in this case.

Peer-review: Externally peer-reviewed.

Author Contributions: Concept - C.T., E.K., T.A., I.K., H.K., Ö.G.; Design - E.K., T.A., I.K., H.K., Ö.G.; Supervision - E.K., T.A., I.K., H.K., Ö.G.; Funding - C.T., T.A.; Materials - Ö.G., I.K.; Data Collection and/or Processing - C.T., T.A.; Analysis and/or Interpretation - E.K., T.A.; Literature Review - C.T.; Writer - C.T., E.K.; Critical Review - T.A., I.K., Ö.G.

Conflict of Interest: No conflict of interest was declared by the authors.

Financial Disclosure: The authors declared that this study has received no financial support.

\section{REFERENCES}

1. Handa P, Crowther M, Douketis JD. Portal vein thrombosis: a clinician-oriented and practical review. Clin Appl Thromb Hemost 2014; 20: 498-506. [CrossRef]

2. Sulu B, Demir E, Günerhan Y. A rare cause of recurring abdominal pain after appendectomy in a young patient:
Portal vein thrombosis. Ulus Cerrahi Derg 2012; 28: 42-45. [CrossRef]

3. İnan M, Tansel Sarıoğlu T, Serhat TH. Porto mesenteric venous thrombosis as a rare cause of acute abdomen in a young patient: What should be the process of diagnosis and management? Ulus Cerrahi Derg 2013; 29: 84-87.

4. Uysal E, Çevik E, Çınar O, Acar Y.A, Gök M, Arslan D. Rare cause of abdominal pain at emergency department: portal vein thrombosis. JAEMCR 2011; 2: 17-19. [CrossRef]

5. Çayır K, Çadırcı K, Bilici M, Tekin SB, Keleş M, Emre H. Factor V Leiden mutation induced portal vein thrombosis: Case report. Haseki Tıp Bülteni. 2009; 47: 181-182.

6. Tchuenbou J, Bacq Y, Fimbel B, Metman EH. Portal vein thrombosis associated with factor $\mathrm{V}$ Leiden mutation in a woman who underwent exchange transfusion at birth. Gastroenterol Clin Biol 2003; 27: 645-647.

7. Chawla Y, Duseja A, Dhiman RK. Review article: the modern management of portal vein thrombosis. Aliment Pharmacol Ther 2009; 30: 881-894. [CrossRef]

8. Finsterer J, Stöllberger C, Schäffl-Doweik L. Neurofibromatosis type I and anti-phospholipid antibody syndrome: report of one case. Rev Med Chil 2013; 141: 1068-1071.[CrossRef] 